\title{
Earthquake Early Warning systems
}

Intense ground motion following an earthquake represents a key hazard to the large number of people living in earthquake-prone regions, and can cause substantial damage to roads, buildings and other infrastructure. Providing accurate and reliable Earthquake Early Warning (EEW) alerts prior to the most intense ground shaking would help minimize damage and loss of life. While several algorithms, which use early waveform observations (such as peak amplitudes) to predict later shaking events, have been developed as EEW

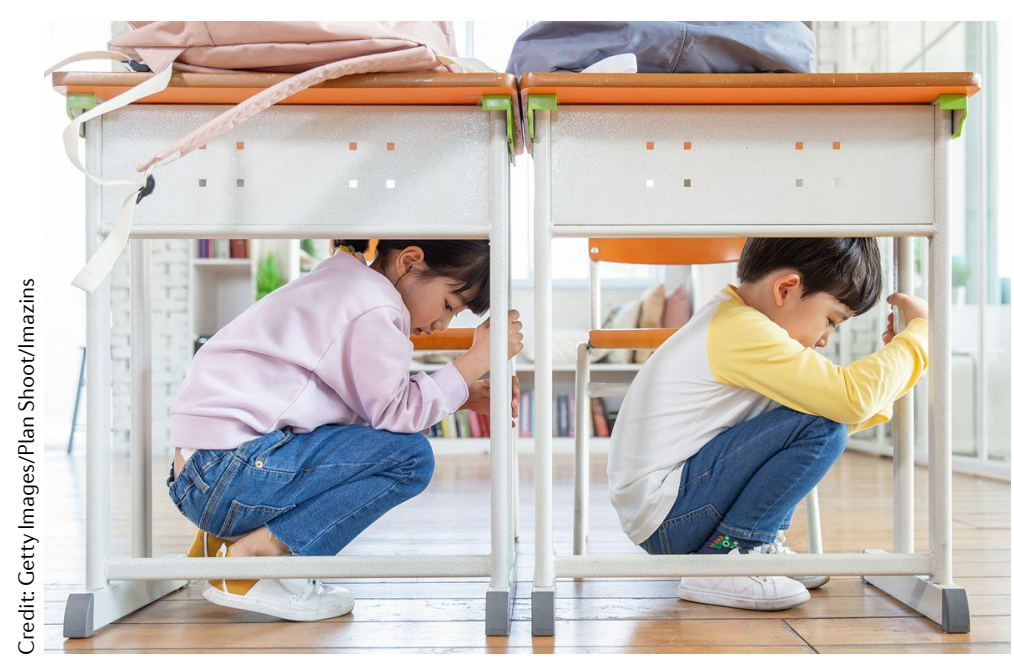

systems, their capabilities have yet to be tested in real time and in response to physical seismic and ground motion data.

\section{Men-Andrin Meier from} California Institute of Technology, USA, and colleagues investigate the capabilities of three EEW algorithms (EPIC, FinDer and PLUM). Using these algorithms, data from strongmotion seismometers (which measure ground acceleration) were 'replayed' and analysed for 219 earthquakes in Japan from 1996 to $2017(\mathrm{Mw}=4.5-9.1)$. They found that all three algorithms have potential as EEW systems. In particular, alerts were triggered at least $5 \mathrm{~s}$ prior to intense shaking for $40-60 \%$ of sites where strong to extreme ground motion occurred, providing enough time to move away from windows or underneath a desk. However, a trade-off exists between the accuracy of predictions and warning time provided. Notably, the PLUM algorithm has the highest percentage of true positive results, but warning times are typically much shorter.

Despite clear progress, EEW algorithms need to be refined to both maximize warning times and minimize the number of false positives. Moreover, future work needs to test whether accurate and timely warnings can be made in regions where motion networks are less dense or homogenous than in Japan. Nevertheless, it is apparent that EEW alert systems can be successfully implemented to reduce the hazards associated with earthquakes.

Matthew Gleeson

ORIGINAL ARTICLE Meier, M.A. et al. How often can Earthquake Early Warning systems alert sites with high intensity ground motion? J. Geophys. Res. https://doi.org/10.1029/2019JB017718 (2020) 